\title{
GIANT CORONARY ANEURYSM IN A 6 WEEK OLD INFANT, AN UNUSUAL FINDING?
}

\author{
Jorge Palacios Argueta ${ }^{1}$, Judith Sanchez Manubens ${ }^{1}$, Flavio Zuccarino ${ }^{2}$, Silvia Teodoro \\ Marin $^{1}$, and Fredy Prada Martinez ${ }^{2}$ \\ ${ }^{1}$ Consorcio Corporacion Sanitaria Parc Tauli \\ ${ }^{2}$ Hospital Sant Joan de Deu
}

June 11, 2021

\begin{abstract}
Kawasaki disease (KD) is a self-limited vasculitis with significant morbidity and even mortality if not treated early. The diagnosis and timely treatment in children younger than 3 months is challenging, most of them have an incomplete or atypical presentation. Coronary artery abnormalities are frequent in this type of patients. We present a 6 -week-old female infant with Kawasaki disease who developed a giant coronary aneurysm. The timely diagnosis and promptly treatment as well as the echocardiographic and multimodality follow-up allowed us to improve our clinical approach and management.
\end{abstract}

TITTLE.

GIANT CORONARY ANEURYSM IN A 6 WEEK OLD INFANT, AN UNUSUAL FINDING?

Palacios Argueta Jorge Roberto MD., (1,A,?) . Sanchez Manubens Judith MD., PhD., (2,A,?,3,) Zuccarino Flavio (5, $\pi, D$, ) MD., PhD.,. Teodoro Marin Silvia (1,A, ?) MD., Prada Martinez Freddy H MD., (4,C,).

Pediatric Cardiology Unit. (1) Pediatric Medicine Service (A). Corporacion Sanitaria del Parc Tauli, Sabadell, Universitat Autónoma de Barcelona. Barcelona, Spain (?).

Pediatric Rheumatology Unit (2). Pediatric Medicine Service (A) . Corporacion Sanitaria del Parc Tauli. Sabadell, Associate professor of Pediatrics, Universitat Autónoma de Barcelona. Barcelona, Spain (?). Pediatric Rheumatology Service (3). Hospital Sant Joan de Deu, Universitat de Barcelona. Barcelona, Spain () .

Pediatric Cardiology Service (4). Hosital Sant Joan de Deu, Universitat de Barcelona. Barcelona, Spain (). Department of Radiology (5). Hospital Sant Joan de Deu, Univesitat de Barcelona, Barcelona, Spain (). Department of Radiology, Head of Cardio-Thoracic Radiology section, Hospital del Mar. Barcelona, Spain (D). Associate professor of Radiology, Universidad Autonoma de Barcelona. Barcelona, Spain $(\pi)$.

Corresponding Author: Jorge Roberto Palacios Argueta. Pediatric Cardiology Unit.Pediatric Medicine Service. Corporacion Sanitaria del Parc Tauli, Sabadell, Universitat Autónoma de Barcelona. Barcelona, Spain. Tel. 00 (34)937231010. Ext. 21006. E-mail: jrpalacios@tauli.cat

\section{KEY WORDS.}

Kawasaki disease. coronary aneurysms. Infant. Fever.

ABSTRACT.

Kawasaki disease $(\mathrm{KD})$ is a self-limited vasculitis with significant morbidity and even mortality if 
not treated early. The diagnosis and timely treatment in children younger than 3 months is challenging, most of them have an incomplete or atypical presentation. Coronary artery abnormalities are frequent in this type of patients. We present a 6 -week-old female infant with Kawasaki disease who developed a giant coronary aneurysm. The timely diagnosis and promptly treatment as well as the echocardiographic and multimodality follow-up allowed us to improve our clinical approach and management.

\section{INTRODUCTION.}

Kawasaki disease $(\mathrm{KD})$ is a self-limited acute febrile illness of unknown cause that predominates in children under 5 years of age. When initially described, the potential for coronary artery complications was not appreciated. KD is now the most common cause of acquired heart disease in children in developed countries. (1). Since its first description in Japan in 1967 by Tomisaku Kawasaki, KD has been increasingly reported worldwide. KD is a great concern among predisposed children it is likely to result in grave clinical consequences (2). Diagnosis is based on clinical findings of its signs and symptoms. Complete $\mathrm{KD}$ is diagnosed with $>5$ days of fever and 4 of 5 clinical features, including bilateral conjunctival injection without exudates, polymorphous exanthema, erythematous changes in the lips and the oral cavity, swelling and erythema of the hands and feet, and cervical lymphadenopathy $(>1.5 \mathrm{~cm})$. Incomplete KD should be considered in patients with unexplained fever for $>5$ days and with 2 or 3 of the clinical features of KD. The peak incidence of KD is from 6 months to 2 years of age. However, there are fewer cases in infants younger than 6 months that can be difficult to diagnose because these patients are more likely to have an incomplete clinical presentation ( 3).Approximately 30-50\% of patients with KD developed transient coronary artery dilatation in the acute stage and about $25 \%$ progress to have serious coronary artery abnormalities such as coronary artery aneurysm and ectasia, if left untreated (2). We present the case of a 6 weeks-old female infant who consults to our center due to 12 hour fever and clinical deterioration, and is admitted for suspicion of invasive bacterial infection. During its evolution at pediatric ward, the patient presented clinical signs compatible with KD and progressive changes in the diameter of the left coronary artery was documented during her hospitalization and her follow up.

CASE.

A 6-week-old female infant with 12 hour high fever and irritability was admitted in the Pediatric Ward with the suspicion of bacterial invasive infection. Blood test, Blood culture, 
Urine culture and Lumbar Puncture were performed on the day of admission. The weight: 4130 grams. Height: 53 centimeters. On the $3^{\text {rd }}$ day she presented torpid evolution with marked irritability, persistence of high fever and a generalized morbiliform rash with craniocaudal progression . During the next 2 days, limbs edema, strawberry tongue and nonsecretory ocular redness with right eye predominance was appreciated.

Blood test showed the elevation of acute phase reactants, C Reactive Protein ( CRP) 25 $\mathrm{mg} / \mathrm{dl}$ and Procalcitonin(PCT) $1.2 \mathrm{ng} / \mathrm{ml}$, hypoalbuminemia of $23.5 \mathrm{~g} / \mathrm{dl}$ and platelet 44,000 stand out. Given the clinical and analytical findings and age of the patient a Kawasaki Disease with incomplete presentation (5-day fever +3 clinical criteria: Rash, limbs edema and conjunctivitis) was diagnosed. On day $5^{\text {th }}$ of admission a first dose of IV

Gamma globulins (IVIG) 2g/ kg was administered. An echocardiogram on day 5th ( figure 1). was normal. A slight clinical improvement of the rash after IVIG was observed, but with worsening of blood parameters (plaquetopenia of 9000 and anemia of $7.9 \mathrm{~g} / \mathrm{dL}$ ). Despite the IVIG treatment, the patient persists with fever on the 8th day, so is decided to start with a second dose of IVIG and IV methylprednisolone $30 \mathrm{mg} / \mathrm{kg} / \mathrm{d}$ for 3 days. The acute phase reactants improve, although the fever persisted, so on 12th day It started 3rd line of treatment for Kawasaki Disease with Anakinra (Anti IL-1) $2 \mathrm{mg} / \mathrm{kg} /$ day subcutaneously.

The patient presents analytical improvement with the appearance of significant desquamation on the feet in the second week. But the fever persisted so on day 16 th of admission a new course of methylprednisolone $30 \mathrm{mg} / \mathrm{kg} /$ day by 3 days was administrated and anakinra was increased to $4 \mathrm{mg} / \mathrm{kg} /$ day.

Echocardiogram follow-up was performed every 48 to 72 hours. A progressive dilatation in the diameter of the descendent anterior left coronary artery with an aneurysm formation was diagnosed. The clinical and analytical response to treatment was good and remained afebrile from day 22 of admission. Given a good evolution, the patient was discharged on the 28th day of admission. The $12^{\text {th }}$ day after discharge the patient was admitted again due to worsening of cardiac aneurysms and analytical blood test parameters. Abciximab infusion, IV Methylprednisolone 3 days course, cyclosporine and clopidogrel were started. The treatment with subcutaneous Anakinra (2mg / kg / day) and Aspirin (5mg / kg / day) was maintained. After 5 days in the pediatric intensive care unit and due to clinic and blood test parameters stability the patient was transferred to the pediatric ward. Anticoagulation with enoxaparin was started adjusted according to factor Xa levels. Serial controls were performed with echocardiograms and Electrocardiogram (EKG). They showed an 
increase in the size of the anterior descending left coronary artery with an aneurysm $+11,53$

$\mathrm{Z}$ score and an enlargement from the proximal portion of the left coronary

artery with a $+5.57 \mathrm{Z}$ score . A 12 Lead EKG showed pathological Q waves on the DII, DIII and AVf (predominantly DIII). The patient was asymptomatic the blood test showed normalization of acute phase reactants parameters. The steroids were progressively reduced in the next 15 days and the patient was discharged from the pediatric ward after 14 days of the $2^{\text {nd }}$ admission. On $5^{\text {th }}$ day after discharge a CT Scan was performed ( fig 4).

A development of giant coronary aneurysm from the left anterior descending coronary artery was diagnosed. She has continued her follow up in the outpatient clinic of Pediatric rheumatology and Pediatric cardiology Units and has not required any hospital admissions since then. In the ambulatory controls after 2 months of the second admission the medications were progressively withdrawn. Enoxaparin, Aspirin and Clopidrogel were maintained . Regular echocardiographic follow up has showed a progressive improvement of the aneurysm of the left anterior descending coronary artery. A diagnostic cardiac catheterization was performed after 2 years of diagnosis and the aneurysm of the left anterior descending coronary artery has disappeared (fig. 5), the patient has a normal development and has fulfilled her milestones for her age.

\section{DISCUSSION.}

We present a case of a 6 week old female with diagnosis of complete KD defined as the presence of fever and all clinical criteria $(1,4)$ that developed a resistant form of KD. A resistant KD is defined by failure in the response to the initial therapy with IVIG (5). Our Patient, despite an adequate and timely treatment, developed a giant left anterior descending coronary aneurysm according the 2017 criteria of American Heart Association guidelines.

This classification of coronary involvement is based on raw dimensions and Z score. No involvement: Always Z score $<2$. Dilatation only 2 to $<2.5$; or if initially $<2$, a decrease in Zscore during follow-up $>1$. Small aneurysm $>2.5$ to $<5$. Medium aneurysm: $>5$ to $<10$, and absolute dimension $<8 \mathrm{~mm}$. Large or giant aneurysm $>10$, or absolute dimension $>8(1,6)$. It is evident that recognition of coronary artery lesions is important in $\mathrm{KD}$ for prediction of late complications and treatment modification. (7). The most common complications of KD include coronary artery dilatation and aneurysms caused by coronary artery vasculitis. Patients younger than 3 months of age who did not fulfil the diagnostic criteria have higher coronary involvement and a higher rate of severe coronary artery lesions (8). Our case showed that although a high degree of clinical suspicion, timely diagnosis, promptly and correct treatment with first line, second line and third line treatments, coronary 
artery complications still occur and are worrisome with high morbidity and mortality. This type of clinical scenario reminds us the importance of the multidisciplinary approach as well as the echocardiographic and multimodality imaging follow-up to better characterize this type of lesions, improving the clinical prognosis of this patients in the short, mid, and long term like we have been described in our patient.

FIGURES.

\section{COMPLIANCE WITH ETHICAL STANDARDS.}

Funding: The authors have not declared any specific grant for this research from any funding agency in the public, commercial or not-for-profit sectors.

Competing interests None declared.

Patient consent Parental/guardian consent obtained

\section{AUTHOR CONTRIBUTIONS.}

Jorge Palacios designed the manuscripts, conceptualized, drafted and revised the article. Judith Sanchez Manubens conceptualized and critically revised the article. Flavio Zuccarino, Freddy Prada and Silvia Teodoro approved the manuscript and participated in the imaging collection and interpretation. All authors fully approve the manuscript as submitted.

\section{REFERENCES.}

1. Mc Crindle B, Rowley A, Newburger J, et al: Diagnosis, Treatment, and Long-Term Management of Kawasaki Disease: A Scientific Statement for Health Professionals From the American Heart Association. Circulation. 2017 Apr 25;135(17): e927-e999.

2. Chen S, Dong Y, Galindo Kiuchi M, et al: Coronary artery complication in Kawasaki Disease and the Importance of Early Intervention. JAMA Pediatrics. 2016 Dec 1;170(12):1156-1163.

3. Yoon YM, Yun HW, Kim SH et al; Clinical Characteristics of Kawasaki Disease in Infants Younger than Six Months: Korean Circ J. 2016 Jul;46(4):550-5

4. Audrey Dionne, Jane C. Burns, Nagib Dahdah et al; Treatment intensification in Patients with Kawasaki Disease and Coronary Aneurysm at Diagnosis. Pediatrics 2019; 143 (6): e20183341.

5. Alessandra Marchesi, Isabella Tarissi de Jaobis, Donato Rigante, et al: Kawasaki disease: guidelines of Italian Society of Pediatrics, part-II, treatment of resistant forms and cardiovascular complications, follow-up, lifestyle and prevention of cardiovascular risks. Italian Journal of Pediatrics.( 2018) 44:103 DOI. Org/10.1186/s13052-018-0529-2

6. Carolyn A. Altman Clinical Assessment of coronary arteries in Kawasaki disease: Focus on echocardiographic assessment. Congenital Heart Disease. 2017: 1-5

7. Shani Ma, So Yung Choi, Hyeong Jun Ahn, et al: The importance of Echocardiogram during the Second week of Illness in Children with Kawasaki Disease. The Journal of Pediatrics. doi.org/10.1016/j.peds.2019.10.067.

8. Wei Li, Li Zhang, Ping Huang et al: Clinical features and mid-term follow/up in infants younger than 3 months with Kawasaki disease in a Chinese population. Journal of Paediatrics and Child Health(2018). Doi> 10.1111/jpc. 14233.

\section{FIGURE LEGENDS.}

FIG 1. Trans Thoracic Echocardiogram with a parasternal short-axis view at the level of the aortic valve ( AV), Right ventricle outflow tract ( RVOT), Right Atrium ( RA). Left coronary descending artery ( DA) with normal diameter. Echocardiogram on day $5^{\text {th }}$ 
FIG 2. Trans Thoracic Echocardiogram with a parasternal short-axis view at the level of the aortic valve (AV), Right Atrium ( RA), Right Ventricle Outflow Tract ( RVOT) showed the progressive dilatation of left coronary and left descending coronary artery ( DA) in the serials echocardiograms at the end of second week of illness.

FIG 3. Trans Thoracic Echocardiogram with a parasternal short-axis view at the level of the aortic valve $(\mathrm{AV})$, showed a giant aneurysm of the left anterior descending coronary artery (DA).

FIG 4. Thoracic CT: Volume rendering reformation (A) shows ascending aorta and LAD (both displayed in red). LAD fusiform aneurysm (length of $13 \mathrm{~mm}$ ) affecting proximal and middle LAD, is clearly defined (Asterisk). Short axis reformation (B) perpendicular to LAD aneurysm depicts a maximum transverse diameter of $5.8 \mathrm{~mm}$ (red circle). DA distal to aneurysm has a small-caliber (2.5mm diameter).

FIG 5. Left coronary angiogram at 2 years of age with normalization in diameter of her anterior descending coronary artery.

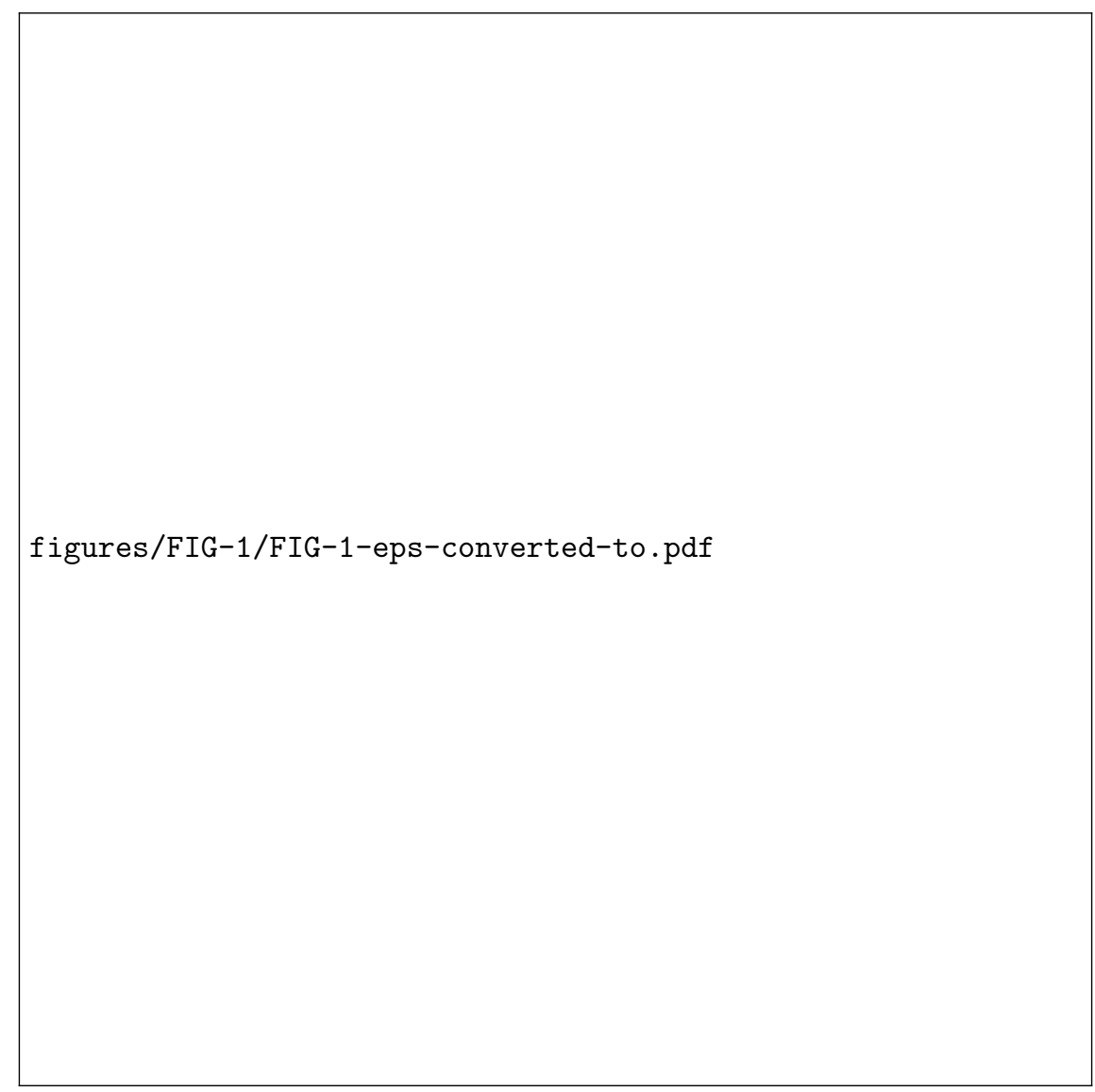


figures/FIG-2/FIG-2-eps-converted-to.pdf 
figures/FIG-3/FIG-3-eps-converted-to.pdf

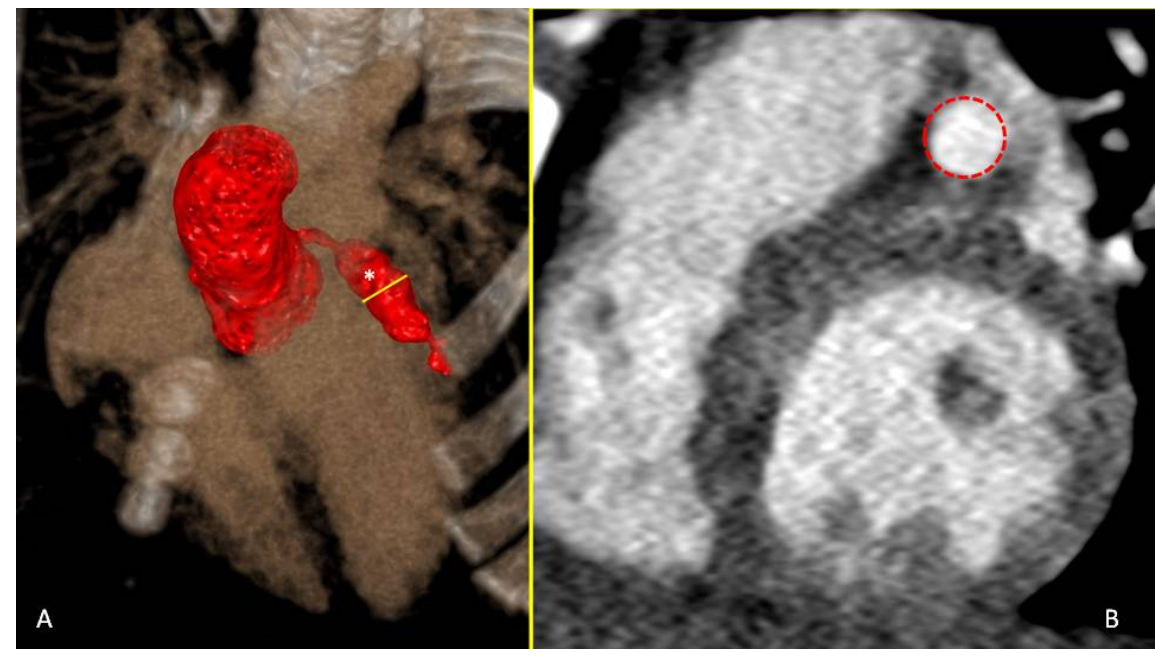




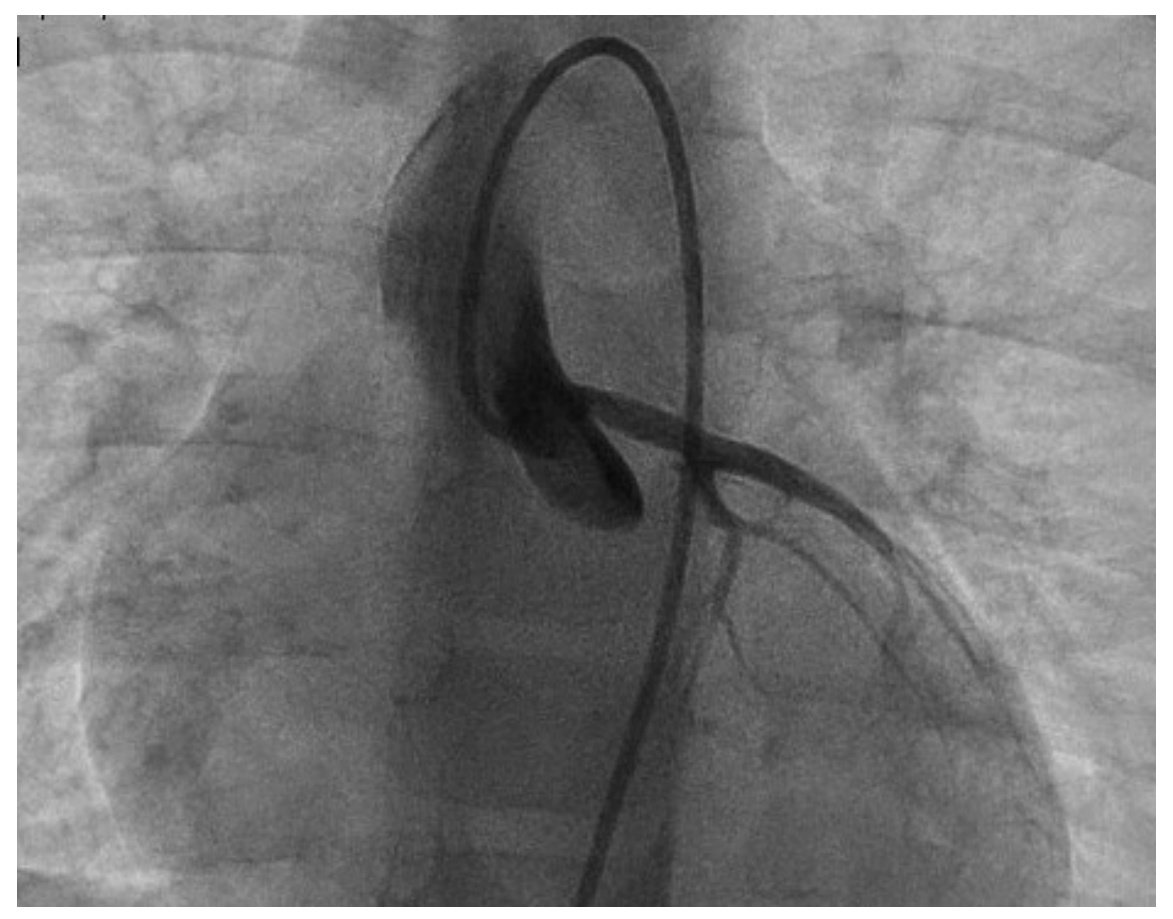

